\title{
DIOPHANTINE TRIPLES WITH VALUES IN THE SEQUENCES OF FIBONACCI AND LUCAS NUMBERS
}

\author{
Florian Luca and Augustine O. Munagi \\ University of the Witwatersrand, South Africa
}

\begin{abstract}
Let $\mathcal{F} L=\{1,2,3,4,5,7,8,11,13,18,21, \ldots\}$ be the set consisting of all Fibonacci and Lucas numbers with positive subscripts. We find all triples $(a, b, c)$ of positive integers $a<b<c$ such that $a b+$ $1, a c+1, b c+1$ are all members of $\mathcal{F} L$.
\end{abstract}

\section{INTRODUCTION}

Let $\mathcal{A}$ be a subset of the positive integers. A Diophantine $m$-tuple with values in $\mathcal{A}$ is a set of $m$ positive integers $\left\{a_{1}, a_{2}, \ldots, a_{m}\right\}$ such that $a_{i} a_{j}+1$ is a member of $\mathcal{A}$ for all $1 \leq i<j \leq m$. The classical case is when $\mathcal{A}$ is the set of squares. The main results here are that $m \leq 5$ and that if there are any examples with $m=5$, then there are only finitely many of them (see [3]). Infinite families of examples are known in this case for $m=4$. Another topic which has received interest is when $\mathcal{A}$ is the set of members of some binary recurrent sequence. Some necessary conditions on the binary recurrence for the existence of infinitely many examples with $m=3$ appear in [4]. For example, one such condition is that the roots of the characteristic equation of the binary recurrence are integers and the smallest one in absolute value is 1 . In particular, for the Fibonacci sequence $\left\{F_{n}\right\}_{n \geq 1}$ given by $F_{1}=F_{2}=1$ and $F_{n+2}=F_{n+1}+F_{n}$ for all $n \geq 1$, and its Lucas companion $\left\{L_{n}\right\}_{n \geq 1}$ given by $L_{1}=1, L_{2}=3, L_{n+2}=L_{n+1}+L_{n}$ for all $n \geq 1$, we have, by the main result in [4], that there are only finitely many Diophantine triples with values in $\mathcal{F}=\left\{F_{n}: n \geq 1\right\}$, and also there are only finitely many Diophantine triples with values in $\mathcal{L}=\left\{L_{n}: n \geq 1\right\}$. In $[5,6]$, it is shown that in fact there is no

2010 Mathematics Subject Classification. 11B39,11D09,11D45,11D75.

Key words and phrases. Diophantine triples, Fibonacci numbers, Lucas numbers. 
Diophantine triple with values in $\mathcal{F}$ and that $\{1,2,3\}$ is the only Diophantine triple with values in $\mathcal{L}$.

Here we take $\mathcal{F} L=\mathcal{F} \cup \mathcal{L}=\{1,2,3,4,5,7,8,11,13,18,21, \ldots\}$ to be the set of Fibonacci and Lucas numbers with positive subscripts and study Diophantine triples with values in $\mathcal{F} L$. Surprisingly, we find an infinite parametric family of examples in addition to a few sporadic ones. Our result is the following.

THEOREM 1.1. Assume that $1 \leq a<b<c$ are positive integers such that $a b+1, a c+1, b c+1$ are members of $\mathcal{F} L$. Then one of the following holds:

(i) $\{a, b, c\}=\left\{F_{2 k}, L_{2 k+1}, L_{2 k+2}\right\}$ for some positive integer $k$;

(ii) $\{a, b, c\}=\{1,2,3\},\{1,3,4\},\{1,2,6\},\{1,2,10\},\{1,6,33\}$.

As a byproduct of our classification theorem of Diophantine triples with values in $\mathcal{F} L$, we get right-away the following result.

COROLlary 1.2. There are no diophantine quadruples with values in $\mathcal{F} L$.

\section{Preliminary Results}

All the results in this section can be found in [6]. Put

$$
(\alpha, \beta)=\left(\frac{1+\sqrt{5}}{2}, \frac{1-\sqrt{5}}{2}\right)
$$

for the two roots of the Fibonacci and Lucas sequences. Their Binet formulas are

$$
F_{n}=\frac{\alpha^{n}-\beta^{n}}{\alpha-\beta} \quad \text { and } \quad L_{n}=\alpha^{n}+\beta^{n} \quad \text { for all } \quad n \geq 1 .
$$

In particular,

(2.2) $\quad \alpha^{n-2} \leq F_{n} \leq \alpha^{n-1}, \quad \alpha^{n-1} \leq L_{n} \leq \alpha^{n+1} \quad$ hold for all $n \geq 1$

and also

(2.3)

$$
\alpha^{n-2} \leq F_{n}-1 \leq \alpha^{n-1}, \quad \alpha^{n-1} \leq L_{n}-1 \leq \alpha^{n} \quad \text { hold for all } n \geq 6 .
$$

LEMMA 2.1. $\quad$ 1. $F_{n} \leq L_{n}$, and equality holds if and only if $n=1$;

2. $F_{n} L_{n}=F_{2 n}$

3. $L_{n}=F_{n+1}+F_{n-1}$;

4. $L_{n}^{2}-5 F_{n}^{2}=4(-1)^{n}$;

5. $L_{2 n}=L_{n}^{2}-2(-1)^{n}$;

6. $L_{3 n}=L_{n}\left(L_{n}^{2}-3(-1)^{n}\right)$;

7. $F_{3 n}=F_{n}\left(5 F_{n}^{2}+3(-1)^{n}\right)$.

For a prime number $p$ and an integer $m$ we write $\nu_{p}(m)$ for the exponent of $p$ in the factorization of $m$. 
Lemma 2.2. The following divisibility relations hold:

1. $\operatorname{gcd}\left(F_{u}, F_{v}\right)=F_{\operatorname{gcd}(u, v)}$;

2. $\operatorname{gcd}\left(L_{u}, L_{v}\right)= \begin{cases}L_{\operatorname{gcd}(u, v),} & \text { if } \nu_{2}(u)=\nu_{2}(v) ; \\ 2, & \text { if } \nu_{2}(u) \neq \nu_{2}(v) \text { and } 3 \mid \operatorname{gcd}(u, v) ; \\ 1, & \text { otherwise; }\end{cases}$

3. $\operatorname{gcd}\left(F_{u}, L_{v}\right)= \begin{cases}L_{\operatorname{gcd}(u, v),} & \text { if } \nu_{2}(u)>\nu_{2}(v) ; \\ 2, & \text { if } \nu_{2}(u) \leq \nu_{2}(v) \text { and } 3 \mid \operatorname{gcd}(u, v) ; \\ 1, & \text { otherwise. }\end{cases}$

For an integer $m$, we write $E_{m}$ and $E_{m}^{\prime}$ for any member of $\left\{F_{m}, L_{m}\right\}$.

Corollary 2.3. We have

$$
\operatorname{gcd}\left(E_{m}, E_{n}\right) \leq L_{\operatorname{gcd}(m, n)} .
$$

Lemma 2.4. The following formulae hold:

1.

2 .

$$
F_{n}-1=\left\{\begin{array}{lll}
F_{\frac{n-1}{2}} L_{\frac{n+1}{2}}, & \text { if } n \equiv 1 & (\bmod 4) ; \\
F_{\frac{n+1}{2}} L_{\frac{n-1}{2}}, & \text { if } n \equiv 3 & (\bmod 4) ; \\
F_{\frac{n-2}{2}} L_{\frac{n+2}{2}}, & \text { if } n \equiv 2 & (\bmod 4) ; \\
F_{\frac{n+2}{2}} L_{\frac{n-2}{2}}, & \text { if } n \equiv 0 & (\bmod 4) ;
\end{array}\right.
$$

$$
L_{n}-1=\left\{\begin{array}{lll}
5 F_{\frac{n-1}{2}} F_{\frac{n+1}{2}}, & \text { if } n \equiv 1 \quad(\bmod 4) ; \\
L_{\frac{n-1}{2}} L_{\frac{n+1}{2}}, & \text { if } n \equiv 3 \quad(\bmod 4) ; \\
\frac{F_{3 n / 2}}{F_{n n 2}}, & \text { if } n \equiv 2 \quad(\bmod 4) ; \\
\frac{L_{3 n / 2}}{L_{n / 2}}, & \text { if } n \equiv 0 \quad(\bmod 4)
\end{array}\right.
$$

3. The Proof of Theorem 1.1

3.1. The setup. The verification that the triples shown at (i) satisfy the hypothesis of the theorem follows because

$$
\begin{aligned}
F_{2 k} L_{2 k+1}+1 & =F_{4 k+1} \in \mathcal{F} L ; \\
F_{2 k} L_{2 k+2}+1 & =F_{4 k+2} \in \mathcal{F} L ; \\
L_{2 k+1} L_{2 k+2}+1 & =L_{4 k+3} \in \mathcal{F} L .
\end{aligned}
$$

The above formulas follow easily from Lemma 2.4. The verification that the triples shown at (ii) satisfy the hypothesis of the theorem is straightforward. We have to show that if $1<a<b<c$ are integers such that

$$
\begin{aligned}
& a b+1=F_{x}, L_{x} ; \\
& a c+1=F_{y}, L_{y} ; \\
& b c+1=F_{z}, L_{z} ;
\end{aligned}
$$

for some positive integers $x, y, z$, then $\{a, b, c\}$ is like in (i) or (ii) of the theorem. Since the cases when right-hand sides are all Fibonacci and all 
Lucas numbers have been treated in [5,6], respectively, we assume that in the right-hand sides of (3.1) there is at least one Fibonacci number and at least one Lucas number.

3.2. The outline. We split the proof into various steps. Each step reveals some structure of the Diophantine triples with values in $\mathcal{F} L$ provided $z$ is sufficiently large. Each section concludes with a verification of the small cases.

3.3. The very small cases. We checked the case when $z \leq 120$. The only sporadic solutions found in this range are the ones shown at (ii) of the theorem.

3.4. A bound for $z$ in terms of $y$. From now on, we assume that $z>120$. Note that the system (3.1) together with inequalities (2.2) lead to

$$
\alpha^{z+1}>L_{z} \geq b c+1>a c+1 \geq F_{y} \geq \alpha^{y-2}, \quad \text { so } \quad z \geq y-2,
$$

and

$$
\alpha^{2 y+2}>L_{y}^{2}>c^{2}>b c+1 \geq F_{z} \geq \alpha^{z-2}, \quad \text { so } \quad z \leq 2 y+3 .
$$

Hence, $z \in[y-2,2 y+3]$.

In fact, the case $z=y-2$ is not possible. Indeed, if this is the case, then since $z \geq 121$, we have $y \geq 123$, and by Lemma 2.1 , we have

$F_{y-1}+F_{y-3}=F_{z+1}+F_{z-1}=L_{z} \geq b c+1>a c+1 \geq F_{y}=F_{y-1}+F_{y-3}+F_{y-4}$,

giving $F_{y-4}<0$, a contradiction for $y \geq 123$. Thus, $z \in[y-1,2 y+3]$.

Let us record this conclusion.

LEMMA 3.1. We have $z \in[y-1,2 y+3]$.

To continue, we distinguish four cases as follows. Consider each of the statements

$$
a c+1=L_{y}, \quad y \text { even }
$$

and

$$
b c+1=L_{z}, \quad z \text { even }
$$

The four cases correspond to whether both (3.3) and (3.4) hold, or only (3.4) holds but not (3.3), or only (3.3) holds but not (3.4), or none holds. 
3.5. The case when both (3.3) and (3.4) hold. Since $a<b<c$, we get that $y<z$. Further, by Lemma 2.4 and Corollary 2.3, we have

$$
c\left|\operatorname{gcd}(a c, b c)=\operatorname{gcd}\left(L_{y}-1, L_{z}-1\right)\right| \operatorname{gcd}\left(E_{3 y / 2}, E_{3 z / 2}^{\prime}\right),
$$

so

$$
c \leq L_{\operatorname{gcd}(3 y / 2,3 z / 2)}=L_{(3 / 2) \operatorname{gcd}(y, z)}<\alpha^{(3 / 2) \operatorname{gcd}(y, z)+1} .
$$

Clearly, $\operatorname{gcd}(y, z)=z / d$ for some integer $d$. If $d \geq 4$, then, by inequalities (2.3), we have

$$
\alpha^{z-1} \leq L_{z}-1=b c<c^{2}<\alpha^{3 z / d+2} \leq \alpha^{3 z / 4+2},
$$

leading to $z \leq 12$, which is impossible. If $d=3$, then $\operatorname{gcd}(y, z)=z / 3$, therefore $y=z / 3,2 z / 3, z$ or $y \geq(4 / 3) z$. Since $y<z$, the last two cases are impossible.

The case $y=z / 3$ leads, via the fact that $z \leq 2 y+3$ (see Lemma 3.1), so $y \geq(z-3) / 2$, to $z / 3=y \geq(z-3) / 2$, so $z \leq 9$, which is impossible.

The case $y=2 z / 3$ implies that $y$ is a multiple of 4 , so, by Lemma 2.4 , we have

$$
L_{y}-1=L_{2 z / 3}-1=\frac{L_{z}}{L_{z / 3}} \mid L_{z},
$$

so $L_{y}-1$ is in fact coprime to $L_{z}-1$. This shows that $c=1$, a contradiction.

3.6. The case when (3.4) holds but (3.3) doesn't. The argument is similar here. We use again Lemma 2.4 to conclude that for some $\delta \in\{ \pm 1, \pm 2\}$ such that $y \equiv \delta(\bmod 2)$, we have

$$
\begin{aligned}
& c|\operatorname{gcd}(a c, b c)| \operatorname{gcd}\left(E_{y}-1, L_{z}-1\right) \mid 5 \operatorname{gcd}\left(E_{(y-\delta) / 2} E_{(y+\delta) / 2}, E_{3 z / 2}^{\prime}\right) \\
& \mid 5 \operatorname{gcd}\left(E_{(y-\delta) / 2}, E_{3 z / 2}^{\prime}\right) \operatorname{gcd}\left(E_{(y+\delta) / 2}, E_{3 z / 2}^{\prime}\right),
\end{aligned}
$$

so, by Corollary 2.3 , we have

$$
\begin{aligned}
c & \leq 5 L_{\operatorname{gcd}((y-\delta) / 2,3 z / 2)} L_{\operatorname{gcd}((y+\delta) / 2,3 z / 2)} \\
& <\alpha^{5.5+\operatorname{gcd}((y-\delta) / 2,3 z / 2)+\operatorname{gcd}((y+\delta) / 2,3 z / 2)} .
\end{aligned}
$$

Here, we used in addition to estimates (2.2) also that $5<\alpha^{3.5}$. We thus get that, by inequality $(2.3)$,

$$
\alpha^{z-1}<L_{z}-1=b c<c^{2}<\alpha^{11+2 \operatorname{gcd}((y-\delta) / 2,3 z / 2)+2 \operatorname{gcd}((y+\delta) / 2,3 z / 2)},
$$

giving

$$
z<12+2 \operatorname{gcd}((y-\delta) / 2,3 z / 2)+2 \operatorname{gcd}((y+\delta) / 2,3 z / 2) .
$$

By parity reasons (recall that $z$ is even), we get

$$
z \leq 10+2 \operatorname{gcd}((y-\delta) / 2,3 z / 2)+2 \operatorname{gcd}((y+\delta) / 2,3 z / 2) .
$$

Let $D_{ \pm}=\operatorname{gcd}((y \pm \delta) / 2,3 z / 2)$. Note that

$$
\operatorname{gcd}\left(D_{+}, D_{-}\right)|\operatorname{gcd}((y+\delta) / 2,(y-\delta) / 2)|(y+\delta) / 2-(y-\delta) / 2=\delta \mid 2,
$$


and certainly $\operatorname{lcm}\left[D_{+}, D_{-}\right] \mid 3 z / 2$. Thus,

$$
D_{+} D_{-}=\operatorname{lcm}\left[D_{+}, D_{-}\right] \operatorname{gcd}\left(D_{+}, D_{-}\right) \mid 2(3 z / 2)=3 z,
$$

showing that $\min \left\{D_{+}, D_{-}\right\} \leq \sqrt{3 z}$. Thus, there exists $\varepsilon \in\{ \pm 1\}$ such that

$$
z \leq 10+2 \sqrt{3 z}+2 \operatorname{gcd}((y+\varepsilon \delta) / 2,3 z / 2) .
$$

The value of $\varepsilon$ is chosen such that $\operatorname{gcd}((y+\varepsilon \delta) / 2,3 z / 2)=\max \left\{D_{+}, D_{-}\right\}$. We write $\operatorname{gcd}((y+\varepsilon \delta) / 2,3 z / 2)=(3 z) /(2 d)$ with some positive integer $d$. Thus,

$$
z \leq 10+2 \sqrt{3 z}+\frac{3 z}{d} .
$$

If $d \geq 5$, we get

$$
z \leq 10+2 \sqrt{3 z}+\frac{3 z}{5}
$$

giving $z \leq 119$, a contradiction.

If $d=4$, then $4 \mid z$. Thus, $L_{z}-1=L_{3 z / 2} / L_{z / 2}$ and in calculation (3.5) we have $E_{3 z / 2}^{\prime}=L_{3 z / 2}$. Further, $(y+\varepsilon \delta) / 2=3 z \lambda / 8$ for some positive integer $\lambda$. If $\lambda \geq 2$, then, using $y \leq z+1$, we get

$$
\frac{z+3}{2} \geq \frac{y+\varepsilon \delta}{2}=\frac{3 z \lambda}{8} \geq \frac{3 z}{4},
$$

leading to $z \leq 6$, a contradiction. Hence, $(y+\varepsilon \delta)=3 z / 8$. In particular, $\nu_{2}((y+\varepsilon \delta) / 2)<\nu_{2}(3 z / 2)$. Lemma 2.2 shows that

$$
\operatorname{gcd}\left(E_{(y+\varepsilon \delta) / 2}, E_{3 z / 2}^{\prime}\right)=\operatorname{gcd}\left(E_{(y+\varepsilon \delta) / 2}, L_{3 z / 2}\right) \leq 2<\alpha^{2} .
$$

Retracing our steps, we conclude that in the right-hand side of (3.8), the summand $3 z / d$ can be replaced by 4 . Thus, we get

$$
z \leq 14+2 \sqrt{3 z}
$$

giving $z \leq 34$, a contradiction.

If $d \leq 2$, then

$$
\frac{y+\varepsilon \delta}{2} \geq \frac{3 z}{2 d} \geq \frac{3 z}{4},
$$

SO

$$
z+3=(z+1)+2 \geq y+\varepsilon \delta \geq \frac{3 z}{2},
$$

giving $z \leq 6$, a contradiction.

If $d=3$, then $y+\varepsilon \delta=\lambda z$ for some positive integer $\lambda$. The case $\lambda \geq 2$, gives $z+3 \geq(z+1)+2 \geq y+\varepsilon \delta=\lambda z \geq 2 z$, so $z \leq 3$, a contradiction. The case $\lambda=1$, leads to $z=y+\varepsilon \delta$, so $z=y-1, y+1, y+2$ (the case $z=y-2$ is not possible by Lemma 3.1).

Let us treat first the case $z=y+2$. Then $y$ is also even so

$$
a c=F_{y}-1=F_{z-2}-1 \mid F_{z} F_{z-4} .
$$

The last divisibility relation follows from Lemma 2.4. Since

$$
b c=L_{z}-1 \mid F_{3 z},
$$


it follows that

$c\left|\operatorname{gcd}\left(F_{z} F_{z-4}, F_{3 z}\right)\right| \operatorname{gcd}\left(F_{z}, F_{3 z}\right) \operatorname{gcd}\left(F_{z-4}, F_{3 z}\right)\left|F_{z} F_{\operatorname{gcd}(3 z, z-4)}\right| F_{12} F_{z}$.

On the other hand, by Lemma 2.4, we have

$$
c \mid \frac{L_{3 z / 2}}{L_{z / 2}}, \quad \text { or } \quad c \mid \frac{F_{3 z / 2}}{F_{z / 2}},
$$

according to whether $4 \mid z$ or not, respectively. Since $F_{12}=144$ and since $F_{z}=F_{z / 2} L_{z / 2}$ (see Lemma 2.1), we get that

$$
c \mid 144 \operatorname{gcd}\left(F_{z / 2}, \frac{L_{3 z / 2}}{L_{z / 2}}\right) \operatorname{gcd}\left(L_{z / 2}, \frac{L_{3 z / 2}}{L_{z / 2}}\right),
$$

or

$$
c \mid 144 \operatorname{gcd}\left(F_{z / 2}, \frac{F_{3 z / 2}}{F_{z / 2}}\right) \operatorname{gcd}\left(L_{z / 2}, \frac{F_{3 z / 2}}{F_{z / 2}}\right),
$$

according to whether $4 \mid z$ or not, respectively. Note that since $F_{z / 2} \mid F_{3 z / 2}$, $L_{z / 2} \mid L_{3 z / 2}$ and $L_{3 z / 2}^{2}-5 F_{3 z / 2}^{2}= \pm 4$ (see again Lemma 2.1), it follows that the first gcd in (3.9) and the second gcd in (3.10) are 1 or 2 . If $4 \mid z$, then

$$
L_{3 z / 2}=L_{z / 2}\left(L_{z / 2}^{2}-3\right),
$$

by Lemma 2.1, so the second gcd in (3.9) divides 3 . Similarly when 4 does not divide $z$, then

$$
F_{3 z / 2}=F_{z / 2}\left(5 F_{z / 2}^{2}-3\right),
$$

again by Lemma 2.1, so the first gcd in (3.10) also divides 3 . In conclusion, in all cases, we get $c \mid 144 \cdot 6=864$, so $\alpha^{z-1}<L_{z}<c^{2} \leq 864^{2}$, so $z \leq 29$.

Assume now $z=y-1$. Then $y=z+1$. If $a c+1=L_{z+1}$, then

$$
a c+1=L_{z+1}>L_{z}=b c+1,
$$

so $a>b$, a contradiction. Thus, $a c+1=F_{z+1}$. Since $a c=F_{z+1}-1$ and $b c=L_{z}-1=\left(F_{z+1}-1\right)+F_{z-1}\left(\right.$ see Lemma 2.1), we get that $c \mid F_{z-1}$. But also

$$
c\left|L_{z}-1=\frac{E_{3 z / 2}}{E_{z / 2}}\right| F_{3 z} .
$$

Hence, $c\left|\operatorname{gcd}\left(F_{3 z}, F_{z-1}\right)=F_{\operatorname{gcd}(3 z, z-1)}\right| F_{3}=2$, a contradiction.

Assume finally that $z=y+1$. Thus, $y=z-1$. If $a c+1=F_{y}$, we get the same contradiction as before. Namely, $c \mid F_{y}-1=F_{z-1}-1$ and also $c \mid L_{z}-1=F_{z+1}+\left(F_{z-1}-1\right)$, therefore $c \mid F_{z+1}$. But also

$$
c\left|L_{z}-1=\frac{E_{3 z / 2}}{E_{z / 2}}\right| F_{3 z}
$$

so $c\left|\operatorname{gcd}\left(F_{3 z}, F_{z+1}\right)=F_{\operatorname{gcd}(3 z, z+1)}\right| F_{3}=2$, a contradiction. Suppose therefore that $a c+1=L_{y}-1=L_{z-1}-1$. Then $c$ also divides

$$
L_{z}-1=L_{z-2}+\left(L_{z-1}-1\right) \text {, }
$$


so $c\left|L_{z-2}\right| F_{2 z-4}$. Since also $c\left|L_{z}-1\right| E_{3 z / 2} \mid F_{3 z}$, we conclude that $c \mid$ $\operatorname{gcd}\left(F_{2 z-4}, F_{3 z}\right)=F_{\operatorname{gcd}(3 z, 2 z-4)} \mid F_{12}$, so $c \leq 144$. So, $\alpha^{z-1}<L_{z}<c^{2}=144^{2}$, so $z \leq 21$, a contradiction.

3.7. The case when (3.3) holds but (3.4) doesn't. We start by following the argument from the beginning of the previous case. We get the conclusions (3.5) and (3.6) except that in the right-hand side, $y$ and $z$ are swapped:

$$
c<\alpha^{5.5+\operatorname{gcd}\left(\left(z-\delta_{1}\right) / 2,3 y / 2\right)+\operatorname{gcd}\left(\left(z+\delta_{1}\right) / 2,3 y / 2\right)},
$$

which together with

$$
\alpha^{z-2}<F_{z} \leq c^{2}
$$

leads to the slightly weaker analogue of (3.7), namely

$$
z \leq 12+2 \operatorname{gcd}\left(\left(z-\delta_{1}\right) / 2,3 y / 2\right)+2 \operatorname{gcd}\left(\left(z+\delta_{1}\right) / 2,3 y / 2\right)
$$

Here, $\delta_{1} \in\{ \pm 1, \pm 2\}$ is such that $z \equiv \delta_{1}(\bmod 2)$. As before, one of the $\operatorname{numbers} \operatorname{gcd}\left(\left(z+\delta_{1}\right) / 2,3 y / 2\right)$ and $\operatorname{gcd}\left(\left(z-\delta_{1}\right) / 2,3 y / 2\right)$ is at most $\sqrt{3 y}$. Hence, there exists $\varepsilon \in\{ \pm 1\}$ such that if we write

$$
\operatorname{gcd}\left(\left(z+\varepsilon \delta_{1}\right) / 2,3 y / 2\right)=\left(z+\varepsilon \delta_{1}\right) /(2 d)
$$

with some integer $d \geq 1$, then

$$
z \leq 12+\frac{(z+\varepsilon \delta)}{d}+2 \sqrt{3 y}
$$

If $d \geq 2$, then we use the fact that $y \leq z+1$, and get

$$
z \leq 12+\frac{z+2}{2}+2 \sqrt{3(z+1)}
$$

giving $z \leq 93$, a contradiction.

If $d=1$, then $\operatorname{gcd}\left(\left(z+\varepsilon \delta_{1}\right) / 2,3 y / 2\right)=\left(z+\varepsilon \delta_{1}\right) / 2$, so $\lambda\left(z+\varepsilon \delta_{1}\right)=3 y$ holds with some positive integer $\lambda$.

If $\lambda \geq 4$, then

$$
z-2 \leq z+\varepsilon \delta_{1}=3 y / \lambda \leq 3 y / 4 \leq(3 z+3) / 4,
$$

giving $z \leq 11$, a contradiction.

If $\lambda=1$, then $y=\left(z+\varepsilon \delta_{1}\right) / 3 \leq(z+2) / 3$. Since $z \leq 2 y+3$, so $y \geq(z-3) / 2$, we get $(z-3) / 2 \leq y \leq(z+2) / 3$, so $z \leq 13$, a contradiction.

If $\lambda=2$, then $z+\varepsilon \delta_{1}=3 y / 2$, so $z=3 y / 2 \pm 1,3 y / 2 \pm 2$. Furthermore, since in this case $z+\varepsilon \delta_{1}$ is even, it follows that $y$ is a multiple of 4 . Thus,

and

$$
a c=L_{y}-1=\frac{L_{3 y / 2}}{L_{y / 2}},
$$

Here, $A^{\prime} \mid 5$. If $E_{3 y / 2}^{\prime}=F_{3 y / 2}$, then

$$
b c=E_{z}^{\prime}-1=A^{\prime} E_{\left(z+\varepsilon \delta_{1}\right) / 2}^{\prime} E_{\left(z-\varepsilon \delta_{1}\right) / 2}^{\prime}=A E_{3 y / 2}^{\prime} E_{3 y / 2-\varepsilon \delta_{1}}^{\prime} .
$$

$c\left|5 \operatorname{gcd}\left(L_{3 y / 2}, F_{3 y / 2}\right) \operatorname{gcd}\left(L_{3 y / 2}, E_{3 y / 2-\varepsilon \delta}^{\prime}\right)\right| 10 \operatorname{gcd}\left(F_{3 y}, F_{3 y-2 \varepsilon \delta_{1}}\right)\left|10 F_{4}\right| 30$, 
so $\alpha^{z-2} \leq F_{z} \leq c^{2} \leq 900$, so $z \leq 16$, a contradiction. If $E_{3 y / 2}^{\prime}=L_{3 y / 2}$, then

$$
b / a=A^{\prime} L_{y / 2} E_{3 y / 2-\varepsilon \delta_{1}}^{\prime} \geq \alpha^{2 y-5}
$$

Since $z \leq 3 y / 2+2$, we get that $y \geq(2 / 3)(z-2)$, so $b \geq \alpha^{2 y-5} \geq \alpha^{4 z / 3-23 / 3}$. Thus, by inequalities (2.2),

$$
\alpha^{z+1}>L_{z}>b^{2}>\alpha^{8 z / 3-46 / 3}
$$

giving $z<9$, a contradiction.

If $\lambda=3$, then $z=y-1, y+1, y+2$. We use that

$$
c\left|a c=L_{y}-1=E_{3 y / 2} / E_{y / 2}\right| F_{3 y} .
$$

If $z=y+2$, then $z$ is even, so $b c+1=F_{z}$. Hence, $c \mid F_{z}-1=F_{y+2}-1$. But also, by Lemma 2.1, we have

$c \mid L_{y}-1=F_{y+1}+F_{y-1}-1=F_{y+2}-F_{y}+F_{y-1}-1=\left(F_{y+2}-1\right)-F_{y-2}$.

Hence, $c \mid F_{y-2}$. Therefore $c\left|\operatorname{gcd}\left(F_{3 y}, F_{y-2}\right)=F_{\operatorname{gcd}(3 y, y-2)}\right| F_{6}=8$. This shows that $\alpha^{z-2}<F_{z} \leq c^{2} \leq 8^{2}$, so $z \leq 10$, a contradiction.

If $z=y-1$, then $b c=E_{z}^{\prime}-1 \leq L_{y-1}-1<L_{y}-1=a c$, a contradiction.

Finally suppose that $z=y+1$. If $b c+1=F_{z}$, it follows that $b c=F_{y+1}-1$. But also $a c=L_{y}-1=\left(F_{y+1}-1\right)+F_{y-1}$. Hence, $c \mid F_{y-1}$, therefore $c\left|\operatorname{gcd}\left(F_{3 y}, F_{y-1}\right)=F_{\operatorname{gcd}(3 y, y-1)}\right| F_{3}=2$, a contradiction. If $b c+1=L_{z}$, then $b c=L_{y+1}-1$. Since $a c=L_{y}-1$, it follows that $c \mid L_{y+1}-L_{y}=L_{y-1}$, so $c \mid F_{2 y-2}$. Thus, $c\left|\operatorname{gcd}\left(F_{3 y}, F_{2 y-2}\right)=F_{\operatorname{gcd}(3 y, 2 y-2)}\right| F_{12}$. This shows that $\alpha^{z-2}<F_{z} \leq c^{2} \leq 144^{2}$, so $z \leq 22$, a contradiction.

From the last three subsections we conclude that neither (3.3) nor (3.4) holds.

3.8. The case when neither (3.3) nor (3.4) holds. Here we show that the following holds.

LEMMA 3.2. We have $|z-y| \leq 4$.

Since now we know that neither (3.3) and (3.4) hold, we get, by Lemma 2.4, that

$$
\begin{aligned}
& a c=E_{y}-1=A E_{(y-\delta) / 2} E_{(y+\delta) / 2}, \\
& b c=E_{z}^{\prime}-1=A^{\prime} E_{\left(z-\delta_{1}\right) / 2}^{\prime} E_{\left(z+\delta_{1}\right) / 2}^{\prime}
\end{aligned}
$$


where $A, A^{\prime} \in\{1,5\}$ and $\delta, \delta_{1} \in\{ \pm 1, \pm 2\}$ are such that $y \equiv \delta(\bmod 2)$ and $z \equiv \delta_{1}(\bmod 2)$. Hence,

$$
\begin{aligned}
c \quad \mid & \operatorname{gcd}(a c, b c) \mid \operatorname{gcd}\left(A E_{(y-\delta) / 2} E_{(y+\delta) / 2}, A^{\prime} E_{\left(z-\delta_{1}\right) / 2}^{\prime} E_{\left(z+\delta_{1}\right) / 2}^{\prime}\right) \\
& \mid \quad 5 \prod_{\varepsilon, \varepsilon_{1} \in\{ \pm 1\}} \operatorname{gcd}\left(E_{(y+\varepsilon \delta) / 2}, E_{\left(z-\varepsilon_{1} \delta_{1}\right) / 2}^{\prime}\right) \\
& \leq 5 \prod_{\varepsilon, \varepsilon_{1} \in\{ \pm 1\}} L_{\operatorname{gcd}\left((y+\varepsilon \delta) / 2,\left(z-\varepsilon_{1} \delta_{1}\right) / 2\right)} \\
& <5 \alpha^{4+\sum_{\varepsilon, \varepsilon_{1} \in\{ \pm 1\}} \operatorname{gcd}\left((y+\varepsilon \delta) / 2,\left(z-\varepsilon_{1} \delta_{1}\right) / 2\right)} .
\end{aligned}
$$

Hence, by (2.2),

$$
\alpha^{z-2} \leq F_{z}<c^{2}<25 \alpha^{8+2 \sum_{\varepsilon, \varepsilon_{1} \in\{ \pm 1\}} \operatorname{gcd}\left((y+\varepsilon \delta) / 2,\left(z-\varepsilon_{1} \delta_{1}\right) / 2\right)},
$$

which together with the fact that $5^{2}<\alpha^{7}$ gives

$$
z \leq 16+2 \sum_{\varepsilon, \varepsilon_{1} \in\{ \pm 1\}} \operatorname{gcd}\left((y+\varepsilon \delta) / 2,\left(z-\varepsilon_{1} \delta_{1}\right) / 2\right) .
$$

By an argument used before, for each $\varepsilon \in\{ \pm 1\}$ the greatest common divisor of

$$
\operatorname{gcd}\left((y+\varepsilon \delta) / 2,\left(z-\delta_{1}\right) / 2\right) \quad \text { and } \quad \operatorname{gcd}\left((y+\varepsilon \delta) / 2,\left(z+\delta_{1}\right) / 2\right)
$$

divides $\delta_{1}$, therefore 2 , and the least common multiple of the above two numbers is at most $(y+2) / 2$. So, the smallest is at most $\sqrt{y+2} \leq \sqrt{z+3}$. In the same way, for each $\varepsilon_{1} \in\{ \pm 1\}$, one of the numbers

$$
\operatorname{gcd}\left(\left(z+\varepsilon_{1} \delta_{1}\right) / 2,(y-\delta) / 2\right) \quad \text { and } \quad \operatorname{gcd}\left(\left(z+\varepsilon_{1} \delta_{1}\right),(y+\delta) / 2\right)
$$

is at most $\sqrt{z+2}$. Thus, up to changing $\delta$ to $-\delta$ and/or $\delta_{1}$ to $-\delta_{1}$ if needed, we conclude that

$$
z \leq 16+\frac{z-\delta_{1}}{d_{1}}+\frac{z+\delta_{1}}{d_{2}}+2 \sqrt{z+2}+2 \sqrt{z+3} .
$$

Here, $d_{1}$ and $d_{2}$ are defined by

$$
\begin{aligned}
& \operatorname{gcd}\left((y-\delta) / 2,\left(z-\delta_{1}\right) / 2\right)=\left(z-\delta_{1}\right) /\left(2 d_{1}\right) ; \\
& \operatorname{gcd}\left((y+\delta) / 2,\left(z+\delta_{1}\right) / 2\right)=\left(z+\delta_{1}\right) /\left(2 d_{2}\right) .
\end{aligned}
$$

Let us treat first the case when one of $d_{1}, d_{2}$ equals 1 . Say $d_{1}=1$. Then $y-\delta=\lambda\left(z-\delta_{1}\right)$. If $\lambda \geq 2$, we then have $z-2 \leq z-\delta_{1}=(y-\delta) / \lambda \leq(z+3) / 2$, so $z \leq 7$, a contradiction. Thus, $\lambda=1$, so $y-\delta=z-\delta_{1}$. In particular, $|y-z|=\left|\delta-\delta_{1}\right| \leq 4$, which is the title of this section. The same conclusion is reached in the case when $d_{2}=1$.

So, assume that $\min \left\{d_{1}, d_{2}\right\} \geq 2$. Suppose first that $d_{1}=d_{2}=2$. Since $d_{1}=2$, it follows that $(y-\delta)=\lambda\left(z-\delta_{1}\right) / 2$, where $\lambda$ cannot be 2 . If $\lambda \geq 3$, then $z+3 \geq y+2 \geq y-\delta=\lambda\left(z-\delta_{1}\right) / 2 \geq 3(z-2) / 2$, so $z \leq 12$, a contradiction. Thus, $\lambda=1$ and $y-\delta=\left(z-\delta_{1}\right) / 2$, therefore $2 y-z=2 \delta-\delta_{1}$. 
Since also $d_{2}=2$, we get that $y+\delta=\lambda_{1}\left(z+\delta_{1}\right) / 2$, where $\lambda_{1}$ cannot be 2 . A similar argument as before shows that $\lambda_{1}=1$, so $y+\delta=\left(z+\delta_{1}\right) / 2$, so $2 y-z=-\left(2 \delta-\delta_{1}\right)$. Hence, $2 \delta-\delta_{1}=y-2 z=-\left(2 \delta-\delta_{1}\right)$, so $2 \delta=\delta_{1}$. This shows that $2 y=z$. In particular, $y=z / 2>60$. Hence, $z$ is even. In particular, since (3.4) does not hold, we get

$$
b c=F_{2 y}-1 .
$$

If $a c=F_{y}-1$, then $c \leq F_{y}-1$, so

$$
F_{2 y}=1+b c<c^{2}<\left(F_{y}-1\right)^{2},
$$

a contradiction for $y>60$ (in fact, even for $y \geq 2$ ), conclusion which can be reached using the Binet formulas (2.1). Assume that $a c=L_{y}-1$. If $a \geq 2$, then $c \leq\left(L_{y}-1\right) / 2$, so

$$
F_{2 y}=1+b c<c^{2}<\left(L_{y}-1\right)^{2} / 4,
$$

again a contradiction for $y>60$ (in fact, even for $y \geq 2$ ), which can be checked using the Binet formulas (2.1). Hence, $a=1, c=L_{y}-1$, so

$$
L_{y}-1 \mid b c=F_{2 y}-1=F_{y} L_{y}-1=F_{y}\left(L_{y}-1\right)+\left(F_{y}-1\right),
$$

so $L_{y}-1 \mid F_{y}-1$, a contradiction for any $y \geq 3$ because then $1<F_{y}<L_{y}$ (see Lemma 2.1). This shows that it is not possible that $d_{1}=d_{2}=2$. Hence, $\min \left\{d_{1}, d_{2}\right\}=2$ and $\max \left\{d_{1}, d_{2}\right\} \geq 3$. Thus, $1 / d_{1}+1 / d_{2} \leq 5 / 6$. Returning to $(3.13)$, we get

$$
\begin{aligned}
z & \leq 16+(z+2)\left(1 / d_{1}+1 / d_{2}\right)+2 \sqrt{z+2}+2 \sqrt{z+3} \\
& \leq 16+\frac{5(z+2)}{6}+2 \sqrt{z+2}+2 \sqrt{z+3},
\end{aligned}
$$

giving $z<776$. To deal with this last range, we looked at

$$
\begin{aligned}
\mathcal{F} L & =\{1,2,3,4,5,7,8,11,13,18,21, \ldots\} \\
& =\left\{L_{1}, F_{3}, F_{4}, L_{3}, F_{5}, L_{4}, F_{6}, \ldots, F_{n+1}, L_{n}, F_{n+2}, L_{n+1}, \ldots\right\} .
\end{aligned}
$$

Let $\mathcal{F} L_{m}$ be the $m$ th element in $\mathcal{F} L$. Note that since $1 \leq a<b<c$, it follows that $b c+1>a c+1 \geq 4$, so both $a c+1, b c+1$ are in

$$
\mathcal{F} L \backslash\{1,2,3\}=\left\{L_{3}, F_{5}, L_{4}, F_{6}, \ldots, F_{n+2}, L_{n+1}, \ldots\right\} .
$$

The general formulas are

$$
\begin{aligned}
& \mathcal{F} L_{1}=1, \quad \mathcal{F} L_{2}=2, \quad \mathcal{F} L_{3}=3,
\end{aligned}
$$

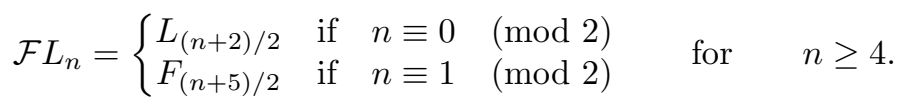

We checked computationally that the only pairs $1<k<n \leq 1600$ with $\operatorname{gcd}\left(\mathcal{F} L_{n}-1, \mathcal{F} L_{k}-1\right\} \geq \sqrt{\mathcal{F} L}_{n}$ which moreover satisfy $k \leq n-7$ are 
$(k, n)=(13+16 t, 20+16 t)$ for $t \in[0,99]$. The difference $n-k$ is always 7 for such pairs. To deal with them, we use a theoretical argument. Note that

$$
\begin{aligned}
& \mathcal{F} L_{k}-1=F_{9+8 t}-1=F_{4 t+4} L_{4 t+5}, \\
& \mathcal{F} L_{n}-1=L_{11+8 t}-1=L_{4 t+6} L_{4 t+5} .
\end{aligned}
$$

Hence,

$$
\begin{aligned}
\operatorname{gcd}\left(\mathcal{F} L_{k}-1, \mathcal{F}_{n}-1\right) & =\operatorname{gcd}\left(F_{4 t+4} L_{4 t+5}, L_{4 t+6} L_{4 t+5}\right) \\
& =L_{4 t+5} \operatorname{gcd}\left(F_{4 t+4}, L_{4 t+6}\right)=3 L_{4 t+5} .
\end{aligned}
$$

The fact that the last gcd above is 3 follows because $3=F_{4}, F_{4} \mid F_{4 t+4}$, $3=L_{2}$ and $L_{2} \mid L_{4 t+6}$ and further,

$$
\operatorname{gcd}\left(F_{4 t+4}, L_{4 t+6}\right)\left|\operatorname{gcd}\left(F_{8 t+8}, F_{8 t+12}\right)=F_{\operatorname{gcd}(8 t+8,8 t+12)}\right| F_{4} .
$$

Thus, $c \mid 3 L_{4 t+5}$. It is not possible that $c \mid L_{4 t+5}$, for if it were so, then

$$
c^{2} \leq L_{4 t+5}^{2}=L_{8 t+10}-2<L_{8 t+11}-1=\mathcal{F} L_{n}-1=b c,
$$

a contradiction. Thus, $c \in\left\{2 L_{4 t+5}, 3 L_{4 t+5}\right\}$. The case $c=2 L_{4 t+5}$ leads to $a=F_{4 t+4} / 2, b=L_{4 t+6} / 2$, so

$$
\begin{aligned}
a b+1 & =\frac{1}{4} F_{4 t+4} L_{4 t+6}+1=\frac{F_{8 t+10}-1}{4}+1=\frac{F_{8 t+10}+3}{4} \\
& =\frac{F_{8 t+10}+F_{4}}{4}=\frac{F_{4 t+3} L_{4 t+7}}{4},
\end{aligned}
$$

while the case $c=3 L_{4 t+5}$ leads to $a=F_{4 t+4} / 3, b=L_{4 t+6} / 3$, so

$$
\begin{aligned}
a b+1 & =\frac{1}{9} F_{4 t+4} L_{4 t+6}+1=\frac{F_{8 t+10}-1}{9}+1=\frac{F_{8 t+10}+8}{9} \\
& =\frac{F_{8 t+10}+F_{6}}{9}=\frac{F_{4 t+8} L_{4 t+2}}{9} .
\end{aligned}
$$

It remains to show that neither $F_{4 t+3} L_{4 t+7} / 4$ nor $F_{4 t+8} L_{4 t+2} / 9$ belongs to $\mathcal{F} L$. This follows right away for $t>1$ because in this case $F_{4 t+3} / 4>$ $1, L_{4 t+2} / 9>1$ and $4 t+8>4 t+7>12$. Thus, if either of

$$
\frac{F_{4 t+3} L_{4 t+7}}{4}=E_{w}, \quad \text { or } \quad \frac{F_{4 t+8} L_{4 t+2}}{9}=E_{w}
$$

holds, then $w>4 t+7>12$ in the first case and $w>4 t+8>12$ in the second case, and by Carmichael's primitive divisor theorem we get that $E_{w}$ has in both cases a prime factor that does not divide the left-hand side of its corresponding equation, which makes the above equalities impossible. For $t=0,1$, we checked by hand and for $t=0$ we do in fact get the sporadic solution $c=3 L_{8 t+5}=33, a=F_{4 t+4} / 3=1$ and $b=L_{4 t+6} / 3=6$. This implies that even in the range $120<z \leq 776$, we have that $|z-y| \leq 4$. 
3.9. $x \in[z-30, y+1]$. Continuing with the argument from the preceding section, we saw that there exist $\delta, \delta_{1} \in\{ \pm 1, \pm 2\}$ such that $y \equiv \delta(\bmod 2)$, $z \equiv \delta_{1}(\bmod 2)$ and $y-\delta=z-\delta_{1}$. Furthermore,

$$
\begin{aligned}
& a c=E_{y}-1=A E_{(y-\delta) / 2} E_{(y+\delta) / 2} ; \\
& b c=E_{z}^{\prime}-1=A^{\prime} E_{\left(z-\delta_{1}\right) / 2}^{\prime} E_{\left(z+\delta_{1}\right) / 2}^{\prime}=A^{\prime} E_{(y-\delta) / 2}^{\prime} E_{\left(y+2 \delta_{1}-\delta\right) / 2}^{\prime} .
\end{aligned}
$$

The three numbers $\left.(y-\delta) / 2,(y+\delta) / 2, y+2 \delta_{1}-\delta\right) / 2$ are distinct except if $\delta=\delta_{1}$. Assume for the moment that $\delta \neq \delta_{1}$.

If $E_{(y-\delta) / 2} \neq E_{(y-\delta) / 2}^{\prime}$, then $\left\{E_{(y-\delta) / 2}, E_{(y-\delta) / 2}^{\prime}\right\}=\left\{F_{(y-\delta) / 2}, L_{(y-\delta) / 2}\right\}$. Further, in this case,

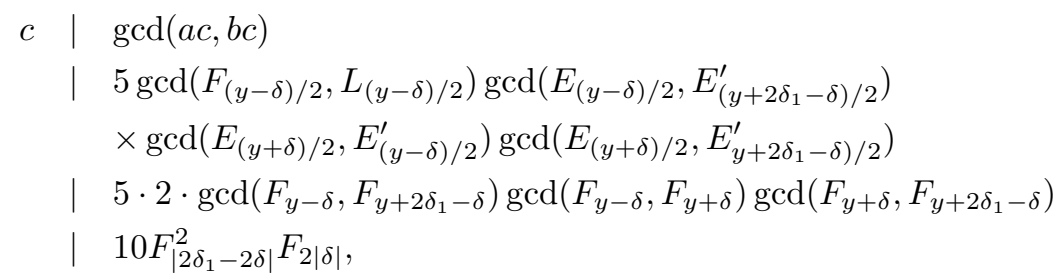

so $c \leq 10 F_{8}^{2} F_{4}=13230$. So, $\alpha^{z-2}<F_{z}<c^{2}<13230^{2}$, therefore $z \leq 42$, a contradiction. So,

$$
E_{(y-\delta) / 2}=E_{(y-\delta) / 2}^{\prime}
$$

But then

$$
\begin{array}{l|l}
c \mid & 5 E_{(y-\delta) / 2} \operatorname{gcd}\left(E_{(y+\delta) / 2}, E_{\left(y+2 \delta_{1}-\delta\right) / 2}^{\prime}\right) \\
\mid & 5 E_{(y-\delta) / 2} \operatorname{gcd}\left(F_{y+\delta}, F_{y+2 \delta_{1}-\delta}\right) \\
\mid & 5 E_{(y-\delta) / 2} F_{\left|2 \delta_{1}-2 \delta\right|}
\end{array}
$$

therefore

$$
c \leq 5 L_{(y-\delta) / 2} F_{8} \leq 105 \alpha^{y / 2+1}<\alpha^{y / 2+11} .
$$

Hence,

$$
a=\frac{E_{y}-1}{c} \geq \frac{F_{y}-1}{\alpha^{y / 2+11}} \geq \alpha^{y / 2-13},
$$

where the last inequality holds by (2.3). Thus,

$$
\alpha^{x+1}>L_{x} \geq a b+1>a^{2}>\alpha^{y-26} \geq \alpha^{z-30},
$$

giving $x \geq z-30$.

This was in case $\delta \neq \delta_{1}$. But if $\delta=\delta_{1}$, then $y=z$, therefore $a c=F_{y}-1$, $b c=L_{y}-1$, and $y$ is odd. Thus, $c \mid F_{y}-1$ and $c \mid L_{y}-1=\left(F_{y}-1\right)+2 F_{y-1}$, showing that $c \mid 2 F_{y-1}$. Since $y$ is odd, $a c=F_{y}-1=E_{(y-1) / 2} E_{(y+1) / 2}$. 
Hence,

$$
\begin{array}{l|l}
c \mid & 2 \operatorname{gcd}\left(F_{y-1}, E_{(y-1) / 2}, E_{(y+1) / 2}\right) \\
\mid & 2 E_{(y-1) / 2} \operatorname{gcd}\left(F_{y-1}, E_{(y+1) / 2}\right) \\
\mid & 2 E_{(y-1) / 2} \operatorname{gcd}\left(F_{y-1}, F_{y+1}\right) \\
\mid & 2 E_{(y-1) / 2}
\end{array}
$$

so

$$
c \leq 2 L_{(y-1) / 2} \leq 2 \alpha^{(y+1) / 2}<\alpha^{y / 2+3} .
$$

So, even in this case when $\delta=\delta_{1}$, we have $c<\alpha^{y / 2+3}<\alpha^{y / 2+11}$, and the previous argument leads to $x \geq z-30$. To see that $x \leq y+1$, assume that this is not so. Then $x \geq y+2$ and

$$
a b=E_{x}-1 \geq F_{y+2}-1 \geq L_{y}-1=b c,
$$

a contradiction. The middle inequality above follows because, by Lemma 2.1, we have $L_{y}=F_{y+1}+F_{y-1}<F_{y+2}$, where the last inequality holds for $y \geq 5$, which is our case since $y=z-\delta_{1}+\delta \geq z-4 \geq 117$. Thus, we have proved the following lemma.

LEMma 3.3. We have $x \in[z-30, y+1]$.

Before closing this section, we record an important byproduct of it, which is the following:

LEMMA 3.4. In the notation

$$
\begin{aligned}
& a c=E_{y}-1=A E_{(y-\delta) / 2} E_{(y+\delta) / 2} ; \\
& b c=E_{z}^{\prime}-1=A^{\prime} E_{\left(z-\delta_{1}\right) / 2}^{\prime} E_{\left(z+\delta_{1}\right) / 2}^{\prime},
\end{aligned}
$$

with the natural conditions $A, A^{\prime} \in\{1,5\}, \delta, \delta_{1} \in\{ \pm 1\}, y \equiv \delta(\bmod 2), z \equiv$ $\delta_{1}(\bmod 2)$, we have

$$
E_{(y-\delta) / 2}=E_{\left(z-\delta_{1}\right) / 2}^{\prime} .
$$

Indeed, this was shown above explicitly when $\delta \neq \delta_{1}$ (see (3.14)), while for $\delta=\delta_{1}$, we have $y=z, E_{y}=F_{y}, E_{z}^{\prime}=L_{z}=L_{y}, y$ is odd, and then the desired conclusion follows from Lemma 2.4 because

$E_{y}-1=F_{y}-1=F_{(y-1) / 2} L_{(y+1) / 2}$ and $E_{z}^{\prime}-1=L_{y}-1=5 F_{(y-1) / 2} F_{(y+1) / 2}$,

or

$E_{y}-1=F_{y}-1=F_{(y+1) / 2} L_{(y-1) / 2}$ and $E_{z}^{\prime}-1=L_{y}-1=L_{(y-1) / 2} L_{(y+1) / 2}$ according to whether $y \equiv 1,3(\bmod 4)$, respectively, hence taking $\delta=1$, we get $E_{(y-\delta) / 2}=E_{\left(z-\delta_{1}\right) / 2}^{\prime}=F_{(y-1) / 2}$ or $L_{(y-1) / 2}$ according to whether $y \equiv 1,3$ $(\bmod 4)$, respectively. 
3.10. The range $z \in[120,1100]$. Now we make a computation to cover the range $120<z \leq 1100$. To do that, we generated all triples $1 \leq l<k<$ $n \leq 2200$ of elements of $\mathcal{F} L$ with the following properties:

(i) $k \in\{n-9, \ldots, n-1\}$;

(ii) $l \in\{n-61, \ldots, k-1\}$;

(iii) $\left(\mathcal{F} L_{k}-1\right)\left(\mathcal{F} L_{l}-1\right)\left(\mathcal{F} L_{n}-1\right)=\square$;

(iv) $\mathcal{F} L_{k}-1 \mid\left(\mathcal{F} L_{l}-1\right)\left(\mathcal{F} L_{n}-1\right)$;

$\mathcal{F} L_{l}-1 \mid\left(\mathcal{F} L_{k}-1\right)\left(\mathcal{F} L_{n}-1\right)$;

$\mathcal{F} L_{n}-1 \mid\left(\mathcal{F} L_{l}-1\right)\left(\mathcal{F} L_{k}-1\right)$.

Indeed, the relevance of the above conditions is that if we write

$$
a b+1=\mathcal{F} L_{k}, \quad a c+1=\mathcal{F} L_{l}, \quad b c+1=\mathcal{F} L_{n},
$$

then (i) follows from the fact that $|z-y| \leq 4$, (ii) comes from the fact that $x \in[z-30, y+1]$, (iii) comes from the fact that

$$
\left(\mathcal{F} L_{l}-1\right)\left(\mathcal{F} L_{k}-1\right)\left(\mathcal{F}_{n}-1\right)=(a b c)^{2},
$$

and (iv) comes from the fact that

$$
\begin{aligned}
& a=\sqrt{\frac{\left(\mathcal{F} L_{k}-1\right)\left(\mathcal{F} L_{l}-1\right)}{\mathcal{F} L_{n}-1},} \\
& b=\sqrt{\frac{\left(\mathcal{F} L_{k}-1\right)\left(\mathcal{F} L_{n}-1\right)}{\mathcal{F} L_{l}-1},} \\
& a=\sqrt{\frac{\left(\mathcal{F} L_{l}-1\right)\left(\mathcal{F} L_{n}-1\right)}{\mathcal{F} L_{k}-1}},
\end{aligned}
$$

are all integers. We got a certain number of possibilities, but all of them came from (i) of the theorem, which are triples of the form

$$
(l, k, n)=(8 t-3,8 t-1,8 t+4)
$$

for some positive integer $t \geq 15$.

Let us now formulate the analogue of (3.3) and (3.4) for the first equation of the system (3.1):

$$
a b+1=L_{x}, \quad x \text { even }
$$

3.11. The case when (3.15) holds. This is very similar to the arguments used in Sections 3.6 and 3.7 so we just recycle those ideas here. Assuming that (3.15) holds then

$$
\begin{aligned}
& a b=L_{x}-1=\frac{E_{3 x / 2}^{\prime \prime}}{E_{x / 2}^{\prime \prime}} \\
& b c=E_{z}-1=A^{\prime} E_{\left(z-\delta_{1}\right) / 2}^{\prime} E_{\left(z+\delta_{1}\right) / 2}^{\prime}
\end{aligned}
$$


giving

SO

$$
\begin{aligned}
& b \quad|\operatorname{gcd}(a b, b c)| \operatorname{gcd}\left(E_{3 x / 2}^{\prime \prime}, A^{\prime} E_{\left(z-\delta_{1}\right) / 2}^{\prime} E_{\left(z+\delta_{1}\right) / 2}^{\prime}\right) \\
& \text { | } 5 \operatorname{gcd}\left(E_{3 x / 2}^{\prime \prime}, E_{\left.\left(z-\delta_{1}\right) / 2\right)}^{\prime}\right) \operatorname{gcd}\left(E_{3 x / 2}^{\prime \prime}, E_{\left.\left(z+\delta_{1}\right) / 2\right)}^{\prime}\right) \text {, }
\end{aligned}
$$

$$
\begin{aligned}
b & \leq 5 L_{\operatorname{gcd}\left(3 x / 2,\left(z-\delta_{1}\right) / 2\right)} L_{\operatorname{gcd}\left(3 x / 2,\left(z+\delta_{1}\right) / 2\right)} \\
& \leq 5 \alpha^{\operatorname{gcd}\left(3 x / 2,\left(z-\delta_{1}\right) / 2\right)+\operatorname{gcd}\left(3 x / 2,\left(z+\delta_{1}\right) / 2\right)+2} .
\end{aligned}
$$

Hence,

$$
\alpha^{x-2}<F_{x}<b^{2}<\alpha^{11+2 \operatorname{gcd}\left(3 x / 2,\left(z-\delta_{1}\right) / 2\right)+2 \operatorname{gcd}\left(3 x / 2,\left(z+\delta_{1}\right) / 2\right)},
$$

where we used again the fact that $5<\alpha^{3.5}$. Hence,

$$
x \leq 12+2 \operatorname{gcd}\left(3 x / 2,\left(z-\delta_{1}\right) / 2\right)+2 \operatorname{gcd}\left(3 x / 2,\left(z+\delta_{1}\right) / 2\right) .
$$

As in Sections 3.6, 3.7, one of $\operatorname{gcd}\left(3 x / 2,\left(z-\delta_{1}\right) / 2\right)$ and $\operatorname{gcd}\left(3 x / 2,\left(z+\delta_{1}\right) / 2\right)$ is at most $\sqrt{3 x}$. So, there is $\varepsilon_{1} \in\{ \pm 1\}$ such that $\operatorname{gcd}\left(3 x / 2,\left(z-\varepsilon_{1} \delta_{1}\right) / 2\right)=3 x / 2 d$ with some positive integer $d$, and further

$$
x \leq 12+3 x / d+2 \sqrt{3 x} .
$$

If $d \geq 4$, then

$$
x \leq 12+3 x / 4+2 \sqrt{3 x},
$$

giving $x \leq 300$, so $z \leq x+30<330$, a contradiction. If $d \leq 2$, then

$$
(z+2) / 2 \geq\left(z-\varepsilon \delta_{1}\right) / 2 \geq 3 x /(2 d) \geq 3(z-30) / 4,
$$

giving $z \leq 94$, a contradiction. If $d=3$, we get $\left(z-\varepsilon \delta_{1}\right) / 2=\lambda x / 2$ for some integer $\lambda \geq 1$. If $\lambda \geq 2$, then $z+2 \geq z-\varepsilon_{1} \delta_{1} \geq 2 x \geq 2(z-30)$, so $z \leq 62$, a contradiction. Finally, if $\lambda=1$, then $z-\varepsilon_{1} \delta_{1}=x$. Thus, $z=x+2, x+1, x-1$. The fact that $z=x-2$ is not possible follows from the argument of Lemma 3.1 which in particular implies that $z \geq x-1$. Now the argument from the end of Section 3.6 (just interchange the pair $(c, y)$ there with the pair $(b, x)$ ) gives $b \leq 864$, so $\alpha^{x-1}<F_{x}<b^{2}<864^{2}$, so $x \leq 30$, a contradiction with $x \geq z-30>1070$.

3.12. All of $x, y, z$ are in an interval of length at most 8. Here, we recycle the ideas of Section 3.8. Write

$$
\begin{gathered}
a b=E_{x}^{\prime \prime}-1=A^{\prime \prime} E_{\left(x-\delta_{2}\right) / 2}^{\prime \prime} E_{\left(x+\delta_{2}\right) / 2}^{\prime \prime} ; \\
b c=E_{z}^{\prime}-1=A^{\prime} E_{\left(z-\delta_{1}\right) / 2}^{\prime} E_{\left(z+\delta_{1}\right) / 2}^{\prime} .
\end{gathered}
$$

Hence,

$$
\begin{aligned}
b \mid & \operatorname{gcd}(a b, b c) \mid \operatorname{gcd}\left(A^{\prime \prime} E_{\left(x-\delta_{2}\right) / 2}^{\prime \prime} E_{\left(x+\delta_{2}\right) / 2}^{\prime \prime}, A^{\prime} E_{\left(z-\delta_{1}\right) / 2}^{\prime} E_{\left(z+\delta_{1}\right) / 2}^{\prime}\right) \\
\mid & 5 \operatorname{gcd}\left(E_{\left(x-\delta_{2}\right) / 2}^{\prime \prime}, E_{\left(z-\delta_{1}\right) / 2}^{\prime}\right) \operatorname{gcd}\left(E_{\left(x-\delta_{2}\right) / 2}^{\prime \prime}, E_{\left(z+\delta_{1}\right) / 2}^{\prime}\right) \\
& \times \operatorname{gcd}\left(E_{\left(x+\delta_{2}\right) / 2}^{\prime \prime}, E_{\left(z-\delta_{1}\right) / 2}^{\prime}\right) \operatorname{gcd}\left(E_{\left(x-\delta_{2}\right) / 2}^{\prime \prime}, E_{\left(z+\delta_{1}\right) / 2}^{\prime}\right)
\end{aligned}
$$


SO

$$
\begin{aligned}
b & \leq 5 \prod_{\varepsilon_{1}, \varepsilon_{2} \in\{ \pm 1\}} L_{\operatorname{gcd}\left(\left(x-\varepsilon_{2} \delta_{2}\right) / 2,\left(z-\varepsilon_{1} \delta_{1}\right) / 2\right)} \\
& \leq \alpha^{7.5+\sum_{\varepsilon_{1}, \varepsilon_{2} \in\{ \pm 1\}} \operatorname{gcd}\left(\left(x-\varepsilon_{2} \delta_{2}\right) / 2,\left(z-\varepsilon_{1} \delta_{1}\right) / 2\right)} .
\end{aligned}
$$

Since

$$
\alpha^{x-2}<F_{x} \leq E_{x}^{\prime \prime}<b^{2}
$$

we get

$$
x \leq 16+2 \sum_{\varepsilon_{1}, \varepsilon_{2} \in\{ \pm 1\}} \operatorname{gcd}\left(\left(x-\varepsilon_{2} \delta_{2}\right) / 2,\left(z-\varepsilon_{1} \delta_{1}\right) / 2\right) .
$$

As in Section 3.8, for each $\varepsilon_{1} \in\{ \pm 1\}$ one of

$$
\operatorname{gcd}\left(\left(z+\varepsilon_{1} \delta_{1}\right) / 2,\left(x-\delta_{2}\right) / 2\right), \quad \text { and } \operatorname{gcd}\left(\left(z+\varepsilon_{1} \delta_{1}\right) / 2,\left(x+\delta_{2}\right) / 2\right)
$$

is at most $\sqrt{z+2}$, while for each $\varepsilon_{2} \in\{ \pm 1\}$, one of

$$
\operatorname{gcd}\left(\left(x+\varepsilon_{2} \delta_{2}\right) / 2,\left(z-\delta_{1}\right) / 2\right), \quad \text { and } \operatorname{gcd}\left(\left(x+\varepsilon_{2} \delta_{2}\right) / 2,\left(z+\delta_{1}\right) / 2\right)
$$

is at most $\sqrt{x+2}$. So, there are $\varepsilon_{1}, \varepsilon_{2} \in\{ \pm 1\}$ such that if we put

$$
\begin{aligned}
& \left.\operatorname{gcd}\left(\left(x+\varepsilon_{2} \delta_{2}\right) / 2, z+\varepsilon_{1} \delta_{1}\right) / 2\right)=\left(z+\varepsilon_{1} \delta_{1}\right) /\left(2 d_{1}\right), \\
& \left.\operatorname{gcd}\left(\left(x-\varepsilon_{2} \delta_{2}\right) / 2, z-\varepsilon_{1} \delta_{1}\right) / 2\right)=\left(z-\varepsilon_{1} \delta_{1}\right) /\left(2 d_{2}\right),
\end{aligned}
$$

then

$$
x \leq 16+\left(z+\varepsilon_{1} \delta_{1}\right) / d_{1}+\left(z-\varepsilon_{1} \delta_{1}\right) / d_{2}+2 \sqrt{x+2}+2 \sqrt{z+2} .
$$

Since, $x \leq z+1$, we get

$$
x \leq 16+\left(z+\varepsilon_{1} \delta_{1}\right) / d_{1}+\left(z-\varepsilon_{1} \delta_{1}\right) / d_{2}+2 \sqrt{z+3}+2 \sqrt{z+2} .
$$

Assume that one of $d_{1}, d_{2}$ equals 1 . Say, $d_{1}=1$. Then $x+\varepsilon_{2} \delta_{2}=\lambda\left(z+\varepsilon_{1} \delta_{1}\right)$ holds with some positive integer $\lambda$. If $\lambda \geq 2$, then

$$
z+3 \geq x+2 \geq x+\varepsilon_{2} \delta_{2}=\lambda\left(z+\varepsilon_{1} \delta_{1}\right) \geq 2(z-2),
$$

so $z \leq 7$, a contradiction. Hence, $\lambda=1$ and $x+\varepsilon_{2} \delta_{2}=z+\varepsilon_{1} \delta_{1}$. In particular $|z-x|=\left|\varepsilon_{2} \delta_{2}-\varepsilon_{1} \delta_{1}\right| \leq 4$. But also $|z-y| \leq 4$, so we get the desired conclusion. This was when $d_{1}=1$. When $d_{2}=1$ a similar argument shows that $x-\varepsilon_{2} \delta_{2}=z-\varepsilon_{1} \delta_{1}$, and we get a the same conclusion that $|z-x| \leq 4$.

It remains to study the case when $\min \left\{d_{1}, d_{2}\right\} \geq 2$. If also $\max \left\{d_{1}, d_{2}\right\} \geq$ 3 , then $1 / d_{1}+1 / d_{2} \leq 5 / 6$, so going back to (3.16) and using that $x \geq z-4$, we get

$$
\begin{aligned}
z-30 \leq x & \leq 16+(z+2)\left(1 / d_{1}+1 / d_{2}\right)+2 \sqrt{z+3}+2 \sqrt{z+2} \\
& \leq 16+5(z+2) / 6+2 \sqrt{z+3}+2 \sqrt{z+2},
\end{aligned}
$$

giving $z<1100$, a contradiction. Finally, assume that $d_{1}=d_{2}=2$. Then $x+\varepsilon_{2} \delta_{2}=\lambda\left(z+\varepsilon_{1} \delta_{1}\right) / 2$ for some integer $\lambda$ which is not 2 . If $\lambda=1$, we then get $z-32 \leq x-2 \leq x+\varepsilon_{2} \delta_{2}=\left(z+\varepsilon_{1} \delta_{1}\right) / 2 \leq(z+2) / 2$, so $z \leq 66$, a contradiction. 
If $\lambda \geq 3$, then $z+3 \geq x+2 \geq x+\varepsilon_{2} \delta_{2} \geq 3\left(z+\varepsilon_{1} \delta_{1}\right) / 2 \geq 3(z-2) / 2$, so $z \leq 12$, again a contradiction.

3.13. Final considerations. Let us now write

$$
\begin{aligned}
a b & =E_{x}^{\prime \prime}-1=A^{\prime \prime} E_{\left(x-\delta_{2}\right) / 2}^{\prime \prime} E_{\left(x+\delta_{2}\right) / 2}^{\prime \prime} ; \\
a c & =E_{y}-1=A E_{(y-\delta) / 2} E_{(y+\delta) / 2} ; \\
b c & =E_{z}^{\prime}-1=A^{\prime} E_{\left(z-\delta_{1}\right) / 2}^{\prime} E_{\left(z+\delta_{1}\right) / 2}^{\prime} .
\end{aligned}
$$

We already saw that $y-\delta=z-\delta_{1}$, that $E_{(y-\delta) / 2}=E_{\left(z-\delta_{1}\right) / 2}^{\prime}$ (see Lemma 3.4) and that up to changing the sign of $\delta_{2}$, there exists $\varepsilon_{1} \in\{ \pm 1\}$ such that $x-\delta_{2}=z-\varepsilon_{1} \delta_{1}$.

Let us show that $E_{\left(x-\delta_{2}\right) / 2}^{\prime \prime}=E_{\left(z-\varepsilon_{1} \delta_{1}\right) / 2}^{\prime}$. Here, we recycle the ideas of Section 3.9.

Consider first the case $\delta_{2} \neq \varepsilon_{1} \delta_{1}$. If $E_{\left(x-\delta_{2}\right) / 2}^{\prime \prime} \neq E_{\left(z-\varepsilon_{1} \delta_{1}\right) / 2}^{\prime}$, we then have $\left\{E_{\left(x-\delta_{2}\right) / 2}^{\prime \prime}, E_{\left(z-\varepsilon_{1} \delta_{1}\right) / 2}^{\prime}\right\}=\left\{F_{\left(x-\delta_{2}\right) / 2}, L_{\left(x-\delta_{2}\right) / 2}\right\}$. Hence,

$b \quad \mid \operatorname{gcd}\left(A^{\prime \prime} E_{\left(x-\delta_{2}\right) / 2}^{\prime \prime} E_{\left(x+\delta_{2}\right) / 2}^{\prime \prime}, A^{\prime} E_{\left(z-\delta_{1}\right) / 2}^{\prime} E_{\left(z+\delta_{1}\right) / 2}^{\prime}\right)$

| $5 \operatorname{gcd}\left(F_{\left(x-\delta_{2}\right) / 2}, L_{\left(x-\delta_{2}\right) / 2}\right) \operatorname{gcd}\left(E_{\left(x-\delta_{2}\right) / 2}^{\prime \prime}, E_{\left(x+2 \varepsilon_{1} \delta_{1}-\delta_{2}\right) / 2}^{\prime}\right)$ $\times \operatorname{gcd}\left(E_{\left(x-\delta_{2}\right) / 2}^{\prime}, E_{\left(x+\delta_{2}\right) / 2}^{\prime \prime}\right) \operatorname{gcd}\left(E_{\left(x+2 \varepsilon_{1} \delta_{1}-\delta_{2}\right) / 2}^{\prime}, E_{\left(x+\delta_{2}\right) / 2}^{\prime \prime}\right)$

| $5 \cdot 2 \operatorname{gcd}\left(F_{x-\delta_{2}}, F_{x+2 \varepsilon_{1} \delta_{1}-\delta_{2}}\right) \operatorname{gcd}\left(F_{x-\delta_{2}}, F_{x+\delta_{2}}\right) \operatorname{gcd}\left(F_{x+2 \varepsilon_{1} \delta_{1}-\delta_{2}}, F_{x+\delta_{2}}\right)$ $\times 10 F_{2\left|\delta_{2}\right|} F_{\left|2 \varepsilon_{1} \delta_{1}-2 \delta_{2}\right|}^{2}$,

so $b \leq 10 F_{4} F_{8}^{2} \leq 30 \times 21^{2} \leq 13230$, giving $\alpha^{x-2}<F_{x} \leq 13230^{2}$, so $x \leq 42$, a contradiction with $x \geq z-8$. This was when $\varepsilon_{1} \delta_{1} \neq \delta_{2}$ and showed that $E_{\left(x-\delta_{2}\right) / 2}^{\prime \prime}=E_{\left(z-\varepsilon_{1} \delta_{1}\right) / 2}^{\prime}$. But if $\varepsilon_{1} \delta_{1}=\delta_{2}$, then $x=z$, so $a b=F_{z}-1, b c=$ $L_{z}-1$ and $z$ is odd. By Lemma 2.4, we have

$$
F_{z}-1=F_{(z-1) / 2} L_{(z+1) / 2}, \quad \text { and } \quad L_{z}-1=5 F_{(z-1) / 2} F_{(z+1) / 2},
$$

and

$$
F_{z}-1=F_{(z+1) / 2} L_{(z-1) / 2}, \quad \text { and } \quad L_{z}-1=L_{(z-1) / 2} L_{(z+1) / 2},
$$

according to whether $z \equiv 1,3(\bmod 4)$, respectively, so it follows that we can take $\delta_{2}=1$ and $E_{\left(x-\delta_{2}\right) / 2}^{\prime \prime}=E_{\left(z+\varepsilon_{1} \delta_{1}\right) / 2}^{\prime}=F_{(z-1) / 2}$ or $L_{(z-1) / 2}$ according to whether $z \equiv 1,3(\bmod 4)$, respectively.

So far we showed that the last of the sets of two elements

$$
\left\{E_{\left(x-\delta_{2}\right) / 2}^{\prime \prime}, E_{\left(x+\delta_{2}\right) / 2}^{\prime \prime}\right\}, \quad\left\{E_{(y-\delta) / 2}, E_{(y+\delta) / 2}\right\}, \quad\left\{E_{\left(z-\delta_{1}\right) / 2}^{\prime}, E_{\left(z+\delta_{1}\right) / 2}^{\prime}\right\}
$$

has a common element with each of the first two. A similar argument shows that the first two sets have a common element. Indeed, just assume that this is not so and recycle the same ideas from Section 3.9 and the beginning of the 
current one with $a b=A^{\prime \prime} E_{\left(x-\delta_{2}\right) / 2}^{\prime \prime} E_{\left(x+\delta_{2}\right) / 2}^{\prime \prime}$ and $a c=A E_{(y-\delta) / 2} E_{(y+\delta) / 2}$ in oder to bound $a$. To bound then $x$ use the fact that

$$
\frac{b}{a}=\frac{b c}{a c}=\frac{E_{z}^{\prime}-1}{E_{y}-1} \leq \frac{L_{z}-1}{F_{y}-1} \leq \frac{\alpha^{z+1}}{\alpha^{y-2}}=\alpha^{z-y+3} \leq \alpha^{7}
$$

(because $z-y \leq 4$ ), to get that $b \leq a \alpha^{7}$, so $\alpha^{x-2} \leq F_{x}<b^{2} \leq \alpha^{14} a^{2}$ to conclude that a small bound on $a$ leads to a small bound on $x$. We do not give the details of such a calculation since it is similar to previous ones and the conclusion is that $x$ is very small (say, $x<100$ ).

Thus, any two of the sets shown at (3.17) have a common element. Further, the product of all the above 6 elements (from the union of the three sets) together with $A A^{\prime} A^{\prime \prime}$ equals $(a b c)^{2}$, which is a perfect square. The next immediate goal is to show that $A=A^{\prime}=A^{\prime \prime}=1$ and that the above 6 elements can be grouped in three equal pairs. Well, the claim that the elements in the union of the sets shown at (3.17) can be grouped in three equal pairs is equivalent to saying that the three sets look like

$$
\{U, V\}, \quad\{V, W\}, \quad\{U, W\}
$$

for some positive integers $U, V, W$. Well, if this would not be so, then the only way that any two of them have a common element is if they are of the form

$$
\{E, X\}, \quad\{E, Y\}, \quad\{E, Z\}
$$

for some positive integers $E, X, Y, Z$. If this is the case and if $p$ is a prime factor of $\operatorname{gcd}(E, X)$, then $p$ divides $\operatorname{gcd}\left(F_{x-\delta_{2}}, F_{x+\delta_{2}}\right) \mid F_{2\left|\delta_{2}\right|}$, so $p$ can only be 3. A similar argument applies to $\operatorname{gcd}(E, Y)$ and $\operatorname{gcd}(E, Z)$. Since then

$$
A A^{\prime} A^{\prime \prime}(E X)(E Y)(E Z)=(a b c)^{2},
$$

we get that $E=\Delta \square$, where $\Delta \in\{1,3,5,15\}$. Since $E=E_{\left(x-\delta_{2}\right) / 2} \in$ $\left\{F_{\left(x-\delta_{2}\right) / 2}, L_{\left(x-\delta_{2}\right) / 2}\right\}$, none of the resulting equations has such large solutions, namely with $x>970$ (see, for example, [1, Theorem 4] for the Fibonacci case and [2, Theorem 2] for the Lucas case). This shows that indeed the sets (3.17) look like (3.18). Then

$$
A A^{\prime} A^{\prime \prime}(U V)(V W)(U W)=(a b c)^{2},
$$

showing that $A A^{\prime} A^{\prime \prime}$ is a square. So, it is 1 or $5^{2}$. Note now that a Fibonacci number among $E_{x}^{\prime \prime}, E_{y}, E_{z}^{\prime}$ has the property that the corresponding expression $E_{x}^{\prime \prime}-1, E_{y}-1, E_{z}^{\prime}-1$ contributes one Fibonacci and one Lucas numbers among the elements from the sets (3.18), so an odd number of Fibonacci and Lucas numbers, whereas Lucas numbers among $E_{x}^{\prime \prime}, E_{y}, E_{z}^{\prime}$ have the property that the corresponding expressions $E_{x}^{\prime \prime}-1, E_{y}-1, E_{z}^{\prime}-1$ contribute an even number of Fibonacci and Lucas numbers $(2$ or 0$)$ to the elements of the sets (3.18). This observation together with the fact that at least one of $E_{x}^{\prime \prime}, E_{y}, E_{z}^{\prime}$ is a Fibonacci number, shows that exactly two of such numbers are Fibonacci numbers and only one is a Lucas number. Thus, by Lemma 
2.4 , two of $A, A^{\prime}, A^{\prime \prime}$ are 1 and the third one is in $\{1,5\}$. Since the product of all three is a square, it follows that $A=A^{\prime}=A^{\prime \prime}=1$. In particular, $U V=a b, V W=a c, U W=b c$, so $\{U, V, W\}=\{a, b, c\}$. Thus, $a, b, c$ are Fibonacci and Lucas numbers. Finally, since two of $E_{x}^{\prime \prime}, E_{y}, E_{z}^{\prime}$ are Fibonacci numbers, and one is a Lucas number, and for the one who is a Lucas number, say $E_{x}^{\prime \prime}$ (just to give an example), we have that $E_{x}^{\prime \prime}-1=A^{\prime \prime} U V$, with $A^{\prime \prime}=1$, it follows that both $U$ and $V$ are Lucas numbers. In conclusion, not only do we infer that $\{a, b, c\}$ are Fibonacci and Lucas numbers, but we learn that exactly two of them are Lucas numbers and one is a Fibonacci number. Finally, since $y-\delta=z-\delta_{1}$ and $z+\delta_{1}=x-\delta_{2}$, we conclude that $a, b, c$ are Fibonacci and Lucas numbers with indices in an interval of length 2. If $\left\{F_{k}, L_{k}\right\} \subset\{a, b, c\}$, then $F_{k} L_{k}+1=F_{2 k}+1$ must be a Fibonacci or a Lucas number, which is false for such large values of $x, y, z$ since

$$
F_{2 k}<F_{2 k}+1<F_{2 k+1} \quad \text { holds for all } \quad k>1 \text {, }
$$

while

$$
L_{2 k-2}<F_{2 k}+1<L_{2 k-1} \quad \text { holds for all } \quad k>2 \text {. }
$$

Hence, $\{a, b, c\}=\left\{F_{u}, L_{v}, L_{w}\right\}$ with distinct indices $u, v, w$ and $\{u, v, w\}=$ $\{t, t+1, t+2\}$. Considering the cases $t=2 k$ and $t=2 k-1$ (so, $t$ even and odd respectively), we only need to analyze the following six possibilities

$$
\begin{aligned}
\{a, b, c\}=\left\{F_{2 k}, L_{2 k+1}, L_{2 k+2}\right\},\left\{L_{2 k}, F_{2 k+1}, L_{2 k+2}\right\},\left\{L_{2 k}, L_{2 k+1}, F_{2 k+2}\right\}, \\
\\
\left\{F_{2 k-1}, L_{2 k}, L_{2 k+1}\right\},\left\{L_{2 k-1}, F_{2 k}, L_{2 k+1}\right\},\left\{L_{2 k-1}, L_{2 k}, F_{2 k+1}\right\} .
\end{aligned}
$$

The first one is the one we want. The remaining five cases can be eliminated right-away by trivial inequalities. For example, for the second triple on the first row above, we have

$$
\max \left\{F_{4 k+3}, L_{4 k+2}\right\}<L_{2 k} L_{2 k+2}+1<\min \left\{F_{4 k+4}, L_{4 k+3}\right\} \quad \text { for all } k \geq 2,
$$

so $L_{2 k} L_{2 k+2}+1$ cannot be a Fibonacci or Lucas number with index at least 997. Similarly,

$$
\begin{gathered}
\max \left\{F_{4 k+2}, L_{4 k+1}\right\}<L_{2 k} L_{2 k+1}+1<\min \left\{F_{4 k+3}, L_{4 k+2}\right\} \quad \text { for all } \quad k \geq 2, \\
\max \left\{F_{4 k+1}, L_{4 k}\right\}<L_{2 k-1} L_{2 k+1}+1<\min \left\{F_{4 k+2}, L_{4 k+1}\right\} \quad \text { for all } \quad k \geq 2, \\
\max \left\{F_{4 k+1}, L_{4 k-1}\right\}<L_{2 k} F_{2 k+1}+1<\min \left\{F_{4 k+2}, L_{4 k}\right\} \quad \text { for all } k \geq 2 .
\end{gathered}
$$

The theorem is proved.

\section{ACKNOWLEDGEMENTS.}

We thank the anonymous referee for a careful reading of the manuscript and for comments which improved the quality of this paper. 


\section{REFERENCES}

[1] Y. Bugeaud, F. Luca, M. Mignotte and S. Siksek, Perfect powers from products of terms in Lucas sequences, J. Reine Angew. Math. 611 (2007), 109-129.

[2] Y. Bugeaud, F. Luca, M. Mignotte and S. Siksek, Almost powers in the Lucas sequence, J. Théor. Nombres Bordeaux 20 (2008), 555-600.

[3] A. Dujella, There are only finitely many Diophantine quintuples, J. Reine Angew. Math. 566 (2004), 183-214.

[4] C. Fuchs, F. Luca and L. Szalay, Diophantine triples with values in binary recurrences, Ann. Sc. Norm. Super. Pisa Cl. Sc. 7 (2008), 579-608.

[5] F. Luca and L. Szalay, Fibonacci Diophantine triples, Glas. Mat. Ser. III 43 (2008), 253-264.

[6] F. Luca and L. Szalay, Lucas Diophantine Triples, Integers 9 (2009), 441-457.

F. Luca

The John Knopfmacher Centre for Applicable Analysis and Number Theory

University of the Witwatersrand

Private Bag X3, Wits 2050

South Africa

E-mail: florian.luca@wits.ac.za

A. O. Munagi

The John Knopfmacher Centre for Applicable Analysis and Number Theory

University of the Witwatersrand

Private Bag X3, Wits 2050

South Africa

E-mail: Augustine.Munagi@wits.ac.za

Received: 16.2.2016.

Revised: 9.7.2016. 